\title{
BREVE ANÁLISIS DEL DELITO DE TRÁFICO DE DROGAS EN LA LEGISLACIÓN PERUANA
}

\author{
Brief ANALYSIS OF DRUG tRAFFICKING CRIME IN PERUVIAN LEGISLATION \\ Meliza Espinoza Hilario \\ Abel Salinas Egoavil \\ Milena Santos Sánchez \\ Anthony Villegas Porras \\ Universidad Continental
}

\section{Resumen}

El presente trabajo trae consigo un análisis del delito del tráfico ilícito de drogas, se abarcan sus antecedentes en la historia del Perú, su legislación actual del tipo, así como dos casos, los cuales servirán como referente para dilucidar los problemas que engloban los atenuantes y agravantes de este delito. Asimismo, podremos identificar los sujetos intervinientes y el bien jurídico protegido, que ayudarán a realizar un análisis normativo con la estructura sistemática de nuestra legislación.

Palabras clave: Tráfico ilícito de drogas; atenuantes; agravantes; producción, comercialización; actos a fines.

\section{Abstract}

The following paper sets an analysis of drug trafficking illicit crime, covering its background in Peru history, its current legislation, as well as two cases which will serve as a reference to elucidate the problems including the mitigating and aggravating factors of this crime. Furthermore, we will be able to identify the intervening subjects and the protected legal right, which will help to carry out a normative analysis with the systematic structure of our legislation.

Palabras clave: Drug trafficking; mitigating circumstances; aggravating circumstances; production circumstances; marketing; commercialization; acts for the purpose. 


\section{ANÁLISIS CRÍTICO DE POLÍTICAS CRIMINALES EXIS- TENTES EN MATERIA DE DROGAS ILÍCITAS}

\subsection{Introducción}

La problemática va centrada a medidas tendientes a reprimir las conductas que favorecen el tráfico ilícito de drogas, tanto a nivel nacional como internacional, así como dar a conocer cuál es el principal problema que aqueja a la sociedad y al Estado peruano. Por ello, el presente análisis va referido al estudio del origen y al planteamiento de la problemática nacional con respecto a la presencia de drogas ilícitas que circulan en nuestro territorio, a dar a conocer al Perú como uno de los principales países que producen cocaína, así como al análisis de la vinculación con la política y dar cuenta de la presencia del narcoterrorismo y su origen.

\subsection{Historia del tráfico ilícito de drogas en el Perú}

Se inicia en la década de 1940, con la presencia de los primeros embarques de cocaína desde el puerto del Callao. En 1985, en el Perú ya se había sembrado 94800 hectáreas de coca, debido al acelerado crecimiento del cultivo de coca en las décadas de 1960 y 1970, que convirtieron al primer lustro de la década de 1980 en el inicio del boom de la comercialización y el auge del procesamiento de la pasta básica de cocaína (PBC). A causa de lo ya mencionado, hay dos consecuencias: en primer lugar, tenemos el incremento del consumo de cocaína en Estados Unidos y, en segundo lugar, la presencia de los cárteles colombianos en Perú.

\subsection{Perú: principal productor de cocaína}

El Perú no es considerado un país de consumidores de drogas ilícitas, esta información se puede corroborar con los datos de una encuesta realizada en el año 2012, en la cual se demuestra que Perú se encuentra debajo de la media que muestra América Latina y se menciona que las personas que han consumido alguna droga ilegal es del $8,1 \%$, mientras que el 4,3\% ha consumido marihuana, la droga más común entre los jóvenes.

A diferencia de otros países de América, donde el porcentaje es mayor y fluctúa entre el 20 y 25 \%, la razón de que en Perú el porcentaje sea bajo es porque se ha instalado una cultura preventiva. Asimismo, se reconoce que 
más del $90 \%$ de la población se encuentra abiertamente en contra de la legalización de las drogas. Esto resulta ser alentador, pero tengamos en cuenta de que Perú es un país productor de PBC, lo que podría crear un contexto en el que los consumidores potenciales podrían adquirirla a precios muy bajos.

El Perú ha sido considerado como uno de los principales productores de droga en el mundo. La Oficina de Naciones Unidas contra la Droga y el Delito reporta que Perú es el segundo productor de cocaína a nivel mundial, ubicándose por debajo de Colombia y por encima de Bolivia. En la actualidad se cultiva coca en 42900 hectáreas del territorio peruano, esto representa al $32 \%$ del cultivo mundial de arbustos de coca. Entre los años 2011 y 2014, esta cifra se habría reducido a 31\%; sin embargo al año siguiente, en el 2015, la cifra volvió a subir.

\subsection{Vinculación con la política}

Esta actividad ilegal no es un problema aislado, ya que también está relacionado e infiltrado en el poder, y sus actores suelen participar en todos los niveles de la política.

Podemos notar con claridad un hecho mencionado en el siglo XX, bajo la presidencia de Alberto Fujimori. La Comisión Especial Investigadora Multipartidaria del Congreso de la República, que era la encargada de investigar y determinar la influencia del narcotráfico en los partidos políticos, reportó que en dicho gobierno se fortalecieron las relaciones entre la política y el narcotráfico, «el mismo que estaba focalizado en un escenario de provisión de drogas, donde la corrupción de militares asentados en las zonas del Alto Huallaga y del VRAEM se daba en el marco de un gobierno de facto manipulado por los servicios de inteligencia cercanos al poder (Montesinos y Fujimori), hecho que era importante para asegurar la salida aérea de las cargas de PBC a los centros de procesamiento de Colombia» (La República).

En la actualidad se han dado a conocer diferentes modalidades de involucramiento de los traficantes de droga en la política y son las siguientes:

- En las campañas y procesos electorales.

- En el proceso de aseguramiento de las rutas locales.

- En el criterio de garantizar diversas formas de impunidad, ejerciendo presiones de nivel político para el favorecimiento en sede policial fiscal y judicial. 
- Presencia y participación en procesos electorales.

- El libre mercado, el impulso de la inversión privada y el favorecimiento de negocios ilícitos.

- Formación de fortunas mal habidas.

- Influencia sobre la policía.

- Subversión, terrorismo y narcoterrorismo.

- Ejercicio de la violencia, sicariato, ajusticiamiento.

\subsection{Narcoterrorismo}

El narcotráfico está fuertemente ligado al terrorismo. En el año 1984, el embajador norteamericano Thumbs en Colombia, denominó a esta relación como «narcoterrorismo». En el Perú, hoy en día se contempla a los narcotraficantes y terroristas en el Alto Huallaga y el valle de los ríos Apurímac, Ene y Mantaro (VRAEM). Anteriormente, a fines de 1980 y 1990, estos grupos tuvieron enfrentamientos. Actualmente, la situación es distinta, conviven e incluso coordinan sus actividades ilícitas con la finalidad de repelar las acciones de las Fuerzas Armadas en dichos territorios.

\subsection{Los delitos de tráfico ilícito de drogas en el ordena- miento peruano}

Respecta de la regulación dentro de nuestra legislación, existen tres momentos en los que el Estado peruano adopta y regula lo referente a la lucha contra las drogas. En primer lugar, desde 1920 se empezó a regular la producción y el comercio de drogas dentro del mercado formal. En segundo lugar, a fines de 1940, el Estado cambió de orientación buscando dar respuesta mediante el Derecho penal al progresivo desarrollo de las organizaciones criminales dedicadas al comercio ilícito de drogas, es decir, la respuesta político-criminal se limitó a lo represivo, a una limitación para frenar tales hechos. Y, en tercer lugar, a fines de 1960, se buscó combinar las medidas legislativas represivas con aquellas que buscaban la salubridad, y es por ello que se empezó a mencionar a las drogas como un problema eminente de índole social.

En nuestro país, la expansión del Derecho penal hacia la represión del tráfico ilícito de drogas se debió a la existencia de nuevas valoraciones de interés prexistentes, y ya en la actualidad se aprecia que el Estado peruano 
ha adecuado su legislación en materia de lucha contra el tráfico ilícito de drogas siguiendo estándares internacionales.

Gracias a estos estos estándares internacionales, el Estado peruano adoptó medidas legislativas orientadas a asegurar la disponibilidad de estupefacientes y sustancias psicotrópicas para fines exclusivamente médicos y científicos, y, además, impuso sanciones penales para combatir la producción, posesión y el tráfico ilícito de drogas.

\subsection{Análisis jurisprudencial: Casación 738-214 Cajamarca}

En el presente caso se cuestiona la sentencia de vista del diecisiete de setiembre de dos mil catorce que confirmó la sentencia de primera instancia del veintiocho de enero de dos mil catorce, en la que se condena a Eliter Sánchez Mera como autor del delito contra la salud pública-posesión de drogas tóxicas para tráfico, en la modalidad agravada del numeral 3 del artículo 297 del Código Penal y además del artículo 296, segundo párrafo, del mismo cuerpo normativo, en agravio del Estado, imponiéndosele 15 años de pena privativa de libertad, diez mil nuevos soles por reparación civil y ciento ochenta días multa e inhabilitación para ejercer cualquier cargo público como profesional técnico de enfermería por el periodo de diez años.

Ya en primera instancia, el juzgado penal colegiado había dictado la sentencia del veintiocho de enero de dos mil catorce, que condena a Eliter Sánchez Mera como autor del delito contra la salud pública-posesión de drogas tóxicas para el tráfico, en agravio del Estado e imponiéndole quince años de sanción y diez mil nuevos soles por reparación civil. Esta decisión tiene como consecuencia de que el abogado defensor interponga un recurso de apelación.

Al llevarse a cabo la audiencia de apelación de sentencia, realizada el veintisiete de agosto de dos mil catorce, la sentencia de vista (recurrida en casación) confirmó la de la primera instancia, que lo condenó como autor del delito indicado.

El Tribunal Supremo da a conocer la intención del uso de esta agravante para incrementar la pena, y va referida al inciso 3 del artículo 297 de que el solo hecho de admitir la imposición de la pena no debe sustentarse o incrementarse en razón de aspectos de la vida o personalidad del autor, esto no vendría a ser parte de un Estado Constitucional de Derecho, por lo que se debió haber tomado dos aspectos importantes para incrementar la pena, 
considerando la agravante. En primer lugar, no basta el solo hecho de que el imputado tenga la condición de "profesional sanitario de salud» $\mathrm{y}$ en segundo lugar, no solo se debe tener en cuenta las intenciones, sino que su accionar delictivo se haya verificado en el contexto, y aprovechado el ejercicio de su condición de tal, con las posibles consecuencias de riesgo para la salud.

Así, se declara fundada la casación de oficio por errónea interpretación del numeral 3 del artículo 297 y artículo 297, y con la consiguiente reformulación, condenaron a Eliter Sánchez Mera como autor del delito contra la posesión de drogas tóxicas para el tráfico, imponiéndosele 8 años de pena privativa de libertad.

\section{DELITOS TíPIFICADOS EN EL ARTíCULO 296}

El artículo 296 del Código penal ha sido considerado como el tipo base del delito del tráfico ilícito de drogas, al definir qué actos configuran dicho delito. Sin embargo, este artículo no tipifica un solo delito, sino cuatro delitos diferentes entre sí.

Es a raíz de esto que cabe considerarlo como un tipo penal de composición múltiple, que además dota de características propias a cada una de las cuatro conductas delictivas, identificándose, por tanto, elementos típicos y momentos consumativos diferenciables.

Ahora bien, a raíz del análisis del artículo 296, encontramos los siguientes delitos: a) De la promoción, favorecimiento o facilitación del consumo ilegal de drogas mediante actos de fabricación o tráfico b) De la posesión de drogas para su tráfico ilícito c) Del suministro, la producción o comercialización de materias primas o insumos destinados a la elaboración de drogas y actos afines de promoción d) De la conspiración para promover, favorecer o facilitar el tráfico ilícito de drogas.

\subsection{De la promoción, favorecimiento o facilitación del consumo ilegal de drogas mediante actos de fabri- cación o tráfico}

En este supuesto, el sujeto activo debe ejecutar actos de fabricación o tráfico y con ello promover, favorecer o facilitar el consumo de drogas a potenciales usuarios. Por lo que se trataría entonces de conductas que difunden 
o expanden el consumo ilegal. Debemos considerar que la base conductual que da origen a todo el ciclo referente a la facilitación del consumo de drogas a terceros es la fabricación y tráfico de las mismas.

En tal sentido, cabe aclarar que se promueve el consumo cuando este no se ha iniciado, se favorece cuando se permite su expansión y se facilita cuando se proporciona la droga a quien ya está iniciado en el consumo. Además, es necesario precisar que este acto punible debe estar siempre orientado hacia el consumo ajeno.

Este supuesto es un tipo penal alternativo, pues comprende varias opciones para la materialización de la conducta punible, aunque para su tipificación tan solo será necesario que el sujeto activo realice cuando menos uno de aquellos comportamientos que constituyen actos de fabricación o tráfico, los cuales podrían ser, según el artículo 89 de la Ley N. 22095: reparar, elaborar, manufacturar, componer, convertir o procesar cualquier sustancia fiscalizada.

Debemos indicar que el tipo subjetivo de este delito requiere el dolo, pues la propia naturaleza del tráfico ilícito de drogas demanda exigir que la motivación del sujeto activo sea lucrativa.

\subsection{De la posesión de drogas para su tráfico ilícito}

Para la consumación de este supuesto delictivo, se requiere que el agente materialice, de cualquier modo, la tenencia o posesión de droga fiscalizada. Cabe mencionar que la clase o cantidad de droga poseída no afecta la tipicidad del acto, pero sí podría configurar una circunstancia atenuante o agravante en la medida que se cumplan con los demás requisititos cuantitativos y cualitativos establecidos en los artículos 297 y 298 del Código penal.

Ha de mencionarse que este supuesto no criminaliza la posesión de drogas para el propio consumo o de posesión de drogas con finalidad diferente a la del tráfico o comercio ilegal, pues carecería de relevancia penal. En un plano subjetivo, la tenencia o posesión dolosa de la droga debe estar orientada hacia un acto posterior del tráfico, es decir, debe coexistir en el agente activo una finalidad comercializadora de la droga poseída. Por esa razón, la tipicidad de este delito exige una tendencia interna trascendente, la cual implica que, además del dolo, el agente activo tenga propuesto un fin ulterior a la posesión y que la droga deba estar destinada al comercio o tráfico ilícito. 
No obstante, ello no requiere que aquel objetivo o finalidad se consume de manera objetiva, pues será suficiente que tal finalidad se encuentre presente al momento de poseer la sustancia delictiva.

\subsection{Del suministro, la producción o comercialización de materias primas o insumos destinados a la elabora- ción de drogas y actos afines de promoción}

En esta estructura normativa, se identifican dos conductas delictivas que se encuentran relacionadas con la introducción al país, la producción, el almacenamiento, el acopio, el suministro o la comercialización de materias primas y sustancias químicas con dos finalidades ilícitas: a) ser destinadas a la elaboración de drogas, y b) su aplicación en las etapas de maceración y procesamiento de materias primas para la obtención de sustancias adictivas fiscalizadoras.

Este tipo penal tipifica de modo autónomo y específico actos que tienen lugar con anterioridad a la fabricación o al tráfico de drogas, aunque también se alude con él a conductas conexas de inducción y colaboración para la realización de tales actividades; tratándose, entonces, de una punición específica de actos preparatorios y formas de participación.

Este tipo penal engloba conductas alternativas, pues tan solo basta que el agente ejecute cualquiera de ellas para que la conducta realizada resulte punible. Entre estas acciones típicas, tenemos:

\subsubsection{Introducción al país}

Son actos de contrabando o importación clandestina, lo cual refiere que las materias primas o sustancias químicas son ingresadas al territorio desde el extranjero por el agente de cualquier manera, en cualquier ocasión o lugar que opere como punto de frontera formal o informal.

\subsubsection{Producción}

Se alude al procedimiento inicial, intermedio o final que corresponda a la producción u obtención de materias primas o sustancias químicas; se infiere que estos son actos anteriores a la provisión de o al acopio, pero que pueden ser realizados secuencialmente por un mismo agente. 


\subsubsection{Acopio}

La conducta del agente equivale a la recolección o concentración física de las materias primas o insumos en un lugar o ante una persona determinada para su posterior desplazamiento a zonas de elaboración de drogas o comercialización de terceros.

\subsubsection{Provisión}

La realización de este supuesto equivale al suministro de materias primas o insumos a terceros para su almacenamiento o acopio, así como para la aplicación de los mismos en los procesos y etapas de producción de drogas.

\subsubsection{Comercialización}

Se trata de cualquier forma de enajenación que haga el agente de las materias primas o insumos. Esta debe pactarse necesariamente en términos lucrativos que le reporten un beneficioso pecuniario.

\subsubsection{Transporte}

Comprende todo tipo de desplazamiento de las materias primas o sustancias químicas que se desarrollen con la finalidad de trasladarlas de un lugar determinado a otro.

\subsubsection{Actos de promoción}

Aquellos que predisponen o favorecen la realización de cualquiera de los actos anteriormente descritos, considerados como formas de inducción o instigación. Cabe mencionar que esta no se puede materializar si existe engaño, amenazas o violencia física.

\subsubsection{Actos de facilitación}

Este supuesto típico involucra cualquier tipo de forma de colaboración, sea material o intelectual, que brinda al agente un tercero que está por realizar actividades de provisión, acopio, producción o comercialización de materias primas o insumos; este se centra en la generación o mantenimiento de condiciones favorables y adecuadas para dichos actos. 


\subsubsection{Actos de financiamiento}

Integrados por las diferentes formas de suministro o facilitación de recursos económicos que deben aplicarse para la iniciación de actividades como: provisión, acopio, producción o comercialización de materias primas o insumos. Este financiamiento puede ser parcial o total, temporal o permanente, sin que ello afecte la tipicidad del acto, aunque no es necesario que la consumación del mismo se realice de manera exitosa.

\subsection{De la conspiración para promover, favorecer o facilitar el tráfico ilícito de drogas}

Es el último delito que incluye el artículo 296, este consiste en participar de una concertación para realizar actos delictivos futuros de promoción, favorecimiento o facilitación de tráfico ilícito de drogas; el mínimo de participantes que deben intervenir en tales actos debe ser dos personas.

Los conspiradores se limitan a idear, bosquejar, acciones o estructuras criminales que se materializan posteriormente, dado que se trata de actos preparatorios criminalizados autónomamente y donde lo esencial y punible es el intercambio y acuerdo de voluntades con relación a un proyecto en común: la promoción, favorecimiento o facilitación del tráfico ilícito de drogas.

Su tipicidad objetiva requiere el dolo, esta se perfecciona con la mera reunión de los conspiradores, aunque esta se relacione una sola vez, por lo que no resulta necesario que el proyecto criminal se implemente materialmente, ni que su concreción sea exitosa o fracase.

\section{CIRCUNSTANCIAS AGRAVANTES Y ATENUANTES}

\subsection{Circunstancias agravantes}

Las circunstancias agravantes se encuentran reguladas en el artículo $297^{\circ}$ del Código penal, están repartidas en 2 grados o niveles para la acreditación de imposición de una mayor penalidad o menor. El segundo nivel conlleva una mayor penalidad que la primera. 


\subsubsection{Circunstancias agravantes de primer nivel o grado}

- La penalidad es no menor de 15 años ni mayor de veinticinco años.

- No menor de ciento ochenta ni mayor de trescientos sesenta y cinco días multa e inhabilitaciones según los incisos 1, 2, 4, 5 y 8 del artículo $36^{\circ}$ del Código penal.

\section{a. Agravante por la condición personal del agente (inciso 1 del ar- tículo $297^{\circ}$ del Código penal)}

El agente comete el hecho abusando del ejercicio de la función pública (...).

Esta agravante vincula al agente con una facultad o un deber especial que tenga, ya sea derivado de su función o profesión ejercida, remitiéndolo a un abuso de la posición o infracción deliberada de los deberes profesionales que ostente la persona.

Además, se debe considerar que el artículo expresamente nos remite a si la persona el la persona que comete el hecho es un funcionario público, pero no es suficiente constatar lo dicho anteriormente, sino que ejerce una situación de abuso.

\section{Inciso 2 y 3 del artículo $297^{\circ}$ del Código penal}

El agente tiene la profesión de educador o se desempeña como tal en cualquiera de los niveles de enseńanza (...).

El agente es médico, farmacéutico, químico, odontólogo o ejerce otra profesión sanitaria (...).

En este caso, la agravante toma solo en cuenta la profesión del infractor y la confianza social que este inspira acerca del ejercicio ético que desempeña. Para este caso, bastará con solo acreditar que el autor o partícipe tenía la calidad de educador o que ejercía una profesión sanitaria al tiempo de la comisión del delito.

Este supuesto es punto de crítica, que a futuro se debería evaluar la posición del agente en el pasado, para subsumir en este inciso, y que en la actualidad existe el vacío sobre una no tipificación cuando el hecho punible sea realizado por un médico que ya no ejerce la profesión o cuando un docente se encuentre jubilado, es de suma importancia ver estas deficiencias. 


\section{b. Agravante por el lugar de comisión del delito (inciso 4 del artí- culo $297^{\circ}$ del Código penal)}

El hecho es cometido en el interior o en inmediaciones de un establecimiento de enseńanza, centro asistencial, de salud, recinto deportivo, lugar de detención o reclusión (...).

Este inciso se encuentra asociado con determinados ambientes o locales, que se encuentren habilitados para una mayor expansión y difusión sobre la facilitación o favorecimiento del consumo ilegal de drogas, «donde exista una mayor peligrosidad que represente la acción criminal en lugares de mayor afluencia de personas, donde el contagio intelectual es más viable y efectivo.» (Prado, 2017, p. 175).

Además, hace alusión a ambientes y locales que se encuentren conectados a actividades educativas, sanitarias, deportivas o de detención y reclusión, donde la afluencia se encuentre activa para la comisión del hecho. No obstante, el agravante no sería de aplicación cuando el agente lo realice, por ejemplo, en horas de la madrugada en la puerta del colegio, o dentro de un centro deportivo que se encuentre clausurado.

\section{c. Agravante por el modo de ejecución (inciso 5 del artículo $297^{\circ}$ del Código Penal)}

...los utiliza para la venta o emplea a una persona inimputable (...).

El segundo párrafo, hace referencia a que el agente utiliza a un intermediario para actos de tráfico ilícito de drogas.

La ley agrava este supuesto, ya que existe un supuesto de autoría mediata, donde el agente utiliza a un menor de edad o a un inimputable para la comisión del hecho delictivo.

De esta manera, el intermediario que realiza esta acción no debe poseer la facultad de comprender el carácter delictuoso de su acto y que sea menor de 18 ańos. La condición de inimputable debe ser de conocimiento del agente, dado que si lo ignora no se aplica esta agravante.

\section{d. Agravante por el destinatario del tráfico ilícito (inciso 5 del artículo $297^{\circ}$ del Código Penal)}

$\mathrm{El}$ agente vende drogas a menores de edad (...). 
El destinatario de las drogas será un menor de edad. La justificación de esta agravante recae en que el destinatario es un menor, donde el consumo lleve a la farmacodependencia precoz, así como los efectos degenerativos a los que llevan estas sustancias.

Se requiere que el agente que cometa este hecho delictivo conozca que sus destinatarios sean de notoria minoría etaria.

Esta agravante no desaparece, aun cuando el menor haya solicitado su venta y posterior consumo.

\section{e. Agravante por la pluralidad de agentes (inciso 6 del artículo $297^{\circ}$ del Código Penal)}

El hecho es cometido por tres o más personas (...).

Se incorpora el agravante cuando la intervención haya sido conjunta de tres o más personas en la comisión delictiva.

Alude a un nivel de coautoría y autoría funcional, el cual debe ser en comisión de un concierto criminal, mas no una banda criminal. Por tanto, la reunión de estos agentes debe dser ocasional y no de una estructuración jerárquica criminal con fines de permanencia y continuidad operativa.

\section{f. Agravante por integrar una organización criminal (inciso 6 del artículo $297^{\circ}$ del Código Penal)}

(...) o en calidad de integrante de una organización criminal dedicada al tráfico ilícito de drogas, o al desvío de sustancias químicas controladas o no controladas o de materias primas a que se refieren los artículos $296^{\circ}$ y $296^{\circ}$-B (...).

El delito debe ser cometido en calidad de integrante de una organización dedicada al tráfico de drogas o que se dedique a la comercialización de insumos para posterior elaboración.

El agente debe haber realizado diversos actos que conduzcan a un hecho delictivo, caso contrario, solo será considerado como integrante de una organización criminal tipificado en el artículo $317^{\circ}$ del Código penal.

El momento donde no se configura un agravante es cuando el agente realice actos delictivos fuera de una estructura criminal, como también si el agente ejerce actos de operación o estrategias. 


\section{g. Agravante por excesiva cantidad de droga (inciso 7 del artículo $297^{\circ}$ del Código penal)}

La droga a comercializarse o comercializada excede las siguientes cantidades: veinte kilogramos de pasta básica de cocaína o sus derivados ilícitos, diez kilogramos de clorhidrato de cocaína, cinco kilogramos de látex de opio o quinientos gramos de sus derivados, y cien kilogramos de marihuana o dos kilogramos de sus derivados o quince gramos de éxtasis, conteniendo Metilendioxianfetamina - MDA, Metilendioximetanfetamina - MDMA, Metanfetamina o sustancias análogas (...).

Este inciso regula las cantidades (volumen) excesivas de droga, que son objeto de la acción delictiva; por ello bastará con superar los parámetros establecidos en el artículo.

- 20 kilogramos de PBC y derivados.

- 10 kilogramos de clorhidrato de cocaína.

- 5 kilogramos de látex de opio.

- 500 gramos de derivados de látex de opio.

- 100 kilogramos de marihuana.

- 2 kilogramos de derivados de marihuana.

- 15 gramos de éxtasis con contenido de metanfetaminas.

Dentro de la norma no se ha establecido la cantidad de materias primas de coca o insumos químicos como el ácido clorhídrico.

\subsubsection{Circunstancias agravantes de segundo nivel o grado}

La penalidad es no menor de veinticinco años ni mayor de treinta y cinco años. Encontrado en el último párrafo del artículo $297^{\circ}$ del Código penal.

\section{a. Agravante por posición del agente en una organización criminal dedicada al tráfico ilícito de drogas (párrafo último del artículo $297^{\circ}$ del Código penal)}

La pena será privativa de libertad no menor de veinticinco ni mayor de treinta y cinco años cuando el agente actúa como jefe, dirigente o cabecilla de una organización dedicada al tráfico ilícito de drogas o insumos para su elaboración (...).

Regula la posición del agente desde tres ámbitos: 
- Jefe,

- dirigente,

- cabecilla.

Haciendo un enfoque, dentro de la organización criminal, podemos definir el jefe o dirigente como aquella persona que tiene el mando central. Este agente se ubica en el punto más alto de la organización.

Este respondería a las preguntas de él qué y el cómo para quien desempeñe el rol de organización, direccionamiento y planes estratégicos y de planeación del grupo delictivo.

En la sociedad se puede reconocer como el capitalista y nexo con organizaciones extranjeras o como el órgano central de nivel ejecutivo y visible de la firma.

Por su parte, el cabecilla es aquel que se encuentra en el mando intermedio, es aquel que se vincula con las planificaciones de las operaciones, control de acciones ejecutivas de otros integrantes de menor nivel, aquel que verifica la cantidad de droga que se compra y su acopio, así como la cantidad de droga que se llevará en los viajes.

\section{b. Agravante por financiamiento a las actividades terroristas (pá- rrafo último del artículo $297^{\circ}$ del Código Penal)}

Igual pena se aplicará al agente que se vale del tráfico ilícito de drogas para financiar actividades terroristas (...).

La coyuntura que involucró regular esta agravante en nuestra legislación fue el hecho de que entre 1980 y 2000 en nuestro territorio peruano las constantes luchas de las organizaciones terroristas se hacían evidentes a la clara obtención de financiamiento voluntario o coactivo de firmas narcotraficantes.

Esta agravante exige que el agente pueda utilizar el acto delictivo para proveer recursos económicos para ejecutar actos u operaciones integrantes de un proyecto terrorista.

El agravante exige que el acto necesariamente no haya sido concretado o ejecutado. 


\subsection{Circunstancias atenuantes}

Se encuentran reguladas en el artículo $297^{\circ}$ del Código penal, tiene una conexión con la cantidad de droga que ejecute el agente. Si este tiene menos de la cantidad establecida, se configura el atenuante; caso contrario, si el agente tiene una mayor cantidad, se configuraría las agravantes correspondientes.

Las penas impuestas serían de no menor de tres ni mayor de siete años de pena privativa de la libertad.

\subsubsection{Atenuante por el volumen de droga que tenga el agen- te (inciso 1 del artículo $298^{\circ}$ del Código penal)}

La cantidad de droga fabricada, extractada, preparada, comercializada o poseída por el agente no sobrepase los cincuenta gramos de pasta básica de cocaína y derivados ilícitos, veinticinco gramos de clorhidrato de cocaína, cinco gramos de látex de opio o un gramo de sus derivados, cien gramos de marihuana o diez gramos de sus derivados o dos gramos de éxtasis, conteniendo Metilendioxianfetamina - MDA, Metilendioximetanfetamina - MDMA, Metanfetamina o sustancias análogas (...).

Refiere a que si su fabricación, extractada, preparada, comercializada o poseída, no exceda las cantidades fijadas por ley. Esto configura una leve intensidad, lo cual favorece a la reducción de la punibilidad del delito.

Las cantidades que configuran atenuantes son:

- 50 gramos de pasta básica de cocaína y derivados ilícitos.

- 25 gramos de clorhidrato de cocaína.

- 5 gramos de látex de opio.

- 1 gramo de derivados de látex de opio.

- 100 gramos de marihuana.

- 10 gramos de derivados de marihuana.

- 2 gramos de éxtasis con contenido de metanfetaminas.

La atenuante no pierde efecto si es que agente realiza los actos delictivos con varias clases de drogas, siempre y cuando respete las cantidades para la atenuación. 


\subsubsection{Atenuantes por volumen de materias primas 0 insumos (inciso 2 del artículo $298^{\circ}$ del Código penal)}

Las materias primas o los insumos comercializados por el agente que no excedan de lo requerido para la elaboración de las cantidades de drogas señaladas en el inciso anterior (...).

De la misma forma, es atenuante el requerir droga con menor cantidad de lo establecido. Por supuesto, estos insumos y materias primas requieren ser verificados por peritos químicos especializados.

\subsection{Irrelevancia de los actos de consumo personal de drogas}

Según el artículo $299^{\circ}$, no es típica la posesión de drogas para el propio consumo. Así el sujeto posea cantidades que superen lo permitido.

\section{EL TRÁFICO ILÍCITO DE DROGAS EN EL PROCESO PENAL}

\subsection{Protección de testigos y peritos}

La experiencia nos ha llevado a darnos cuenta de que, en muchos casos, la incomparecencia o las evasivas de los ciudadanos, ya sea como testigos o como peritos ante causas penales llevadas contra traficantes de drogas, se da por el temor de sufrir represalias, tanto por parte del imputado como por por parte de la organización a la que podría pertenecer.

Por tal motivo, se cae en una situación en la que el acceso a testimonios y pruebas determinantes en estos casos es deficiente y con eso se aumenta la dificultad de llegar a una condena para los que cometen el delito tipificado en el artículo 296 del Código penal.

Es común que, ante casos de tráfico ilícito de drogas, los testigos o peritos que deben comparecer en los juicios para ayudar a la actuación efectiva de la justicia tengan temor por ellos mismos o por sus familias, ya que muchas veces pueden ser amenazados o agredidos para que no lleguen a presentarse en el proceso.

Es por tal motivo que en nuestra legislación se regulan formas de protección para que estos testigos puedan tener seguridad de que no les va a pasar nada malo por su participación en el proceso. 
Para lograr que los testigos o peritos no se vean afectados por su participación en estos casos, tienen acceso a medidas de protección reguladas en los artículos N. 248 y 249 del Código Procesal penal.

\subsection{Análisis de caso}

\section{R.N. $\mathbf{N}^{\circ}$ 1149-2016 Lima Norte}

En base a esta resolución, analizaremos el principio de proporcionalidad en los delitos de tráfico ilícito de drogas.

La sentenciada interpone recurso de nulidad en contra de la resolución que la sentencia a ocho años de pena privativa de libertad efectiva, argumentando que i) en todo momento se consideró responsable de los hechos que se le imputan y aceptando los mismos; ii) no cuenta con antecedentes penales ni policiales; iii) la Sala Penal no consideró estos hechos al momento de emitir el fallo; iv) que, en base a los artículos 160 y 161 del Código procesal penal (confesión sincera), le corresponde una deducción de hasta una tercera parte por debajo del mínimo legal de la penal; v) que, por haberse acogido a la conclusión anticipada, se le debe reducir adicionalmente la pena hasta una sexta parte de la pena conforme al artículo 471 del Código procesal.

Así tal como lo expresa la R.N. N. ${ }^{\circ} 1149-2016$, Lima Norte

[1] as exigencias que determinan la dosificación de la pena no se agotan en los criterios generales de individualización de la pena, sino que también se debe tener en cuenta el principio de proporcionalidad previsto en el artículo octavo del Título Preliminar del Código Penal limite al ius puniendi, que procura la correspondencia entre le injusto cometido y la pena a imponerse. (Principio de Proporcionalidad en el delito de Tráfico Ilícito de Drogas, 2016).

También se debe considerar que el fin que persigue la pena es preventivo, protector y resocializador.

Así, la sentenciada alega que la Sala Superior no tuvo en cuenta su arrepentimiento por el delito cometido y sus condiciones personales y culturales; sin embargo, la Sala Penal Superior sí consideró dichas condiciones. 


\section{BIBLIOGRAFÍA}

Cáceres J. (2008). Habeas corpus contra el autoapertorio de instrucción. Lima: Grijley.

Mendoza, F. (2012). La necesidad de una imputación concreta en el desarrollo del proceso penal cognitivo. Arequipa: San Bernardo.

República, C. d. (s.f.). Narcotráfico y política. 85.

Prado, V. (2017). Derecho penal parte especial: los delitos. Lima: PUCP. 